PROCEEDINGS OF THE

AMERICAN MATHEMATICAL SOCIETY

Volume 129, Number 7 , Pages 1907-1913

S 0002-9939(00)05827-5

Article electronically published on December 4, 2000

\title{
ON CLASSIFICATION OF POLARIZED VARIETIES WITH NON-INTEGRAL NEF VALUES
}

\author{
YICAI ZHAO
}

(Communicated by Ron Donagi)

\begin{abstract}
Let $M$ be an $n$-dimensional normal projective variety with only Gorenstein, terminal, $\mathbb{Q}$-factorial singularities. Let $L$ be an ample line bundle on $M$. Let $\tau$ denote the nef value of $(M, L)$. The classification of $(M, L)$ via the nef value morphism is given for the situations when $\tau$ satisfies $n-3<\tau<n-2$ or $n-4<\tau<n-3$.
\end{abstract}

\section{INTRODUCTION}

Let $M$ be an $n$-dimensional normal projective variety with only $\mathbb{Q}$-Gorenstein, terminal singularities and let $L$ be an ample line bundle on $M . K_{M}$ denotes the canonical divisor, or the canonical sheaf of $M$. Assume that $K_{M}$ is not nef. The nef value of $(M, L)$ is a real number defined as $\tau=\min \left\{r \in \mathbb{R}, K_{M}+r L\right.$ is nef $\}$.

By the Kawamata Rationality Theorem, $\tau$ is a rational number and by the Kawamata-Shokurov Base Point Free Theorem, $\left|m\left(K_{M}+\tau L\right)\right|$ is base point free for $m \gg 0$, and defines a projective surjective morphism $\varphi: M \rightarrow X$ onto a normal variety $X . \varphi$ is called the nef value morphism.

Sommese [S] and Beltrametti and Sommese [BS1] establish and develop the theory of the adjunction theoretic classification of projective varieties. The pairs $(M, L)$ with the nef value $\tau=n-1, n-2$, or $n-2<\tau<n-1$ have been classified in [BS1, $\mathrm{An}]$ and [F2].

This paper studies the pairs $(M, L)$ such that $n-3<\tau<n-2$ or $n-4<$ $\tau<n-3$. We give out the adjunction theoretic classification for these polarized varieties.

\section{Preliminaries}

We work over the complex field $\mathbb{C}$. Throughout this paper, the notions and notations coincide with that in BS1. A variety means an irreducible and reduced projective scheme.

We begin by recalling some facts from adjunction theory and Mori theory. We refer for that to $\mathrm{BS1}$, [KMM] and $[\mathrm{Mo}$.

In this paper, let $M$ be a normal projective variety of dimension $n \geq 2$, and let $L$ be an ample line bundle on $M$.

Received by the editors September 14, 1998 and, in revised form, November 1, 1999.

2000 Mathematics Subject Classification. Primary 14Jxx.

Key words and phrases. Nef value morphism, ample line bundle, projective variety.

(C)2000 American Mathematical Society 
(0.1)Kawamata Rationality Theorem([KMM, (4-1-1)]). Let $M$ be a normal variety of dimension $n$ with terminal singularities and let $r$ be the index of $M$. Let $f: M \rightarrow \mathcal{S}$ be a projective morphism onto variety $\mathcal{S}$. Let $L$ be an $f$-ample line bundle on $M$. If $K_{M}$ is not $f$-nef, then $\tau=\min \left\{r \in \mathbb{R}, K_{M}+r L\right.$ is $f$-nef $\}$ is a positive rational number. Furthermore expressing $r \tau=u / v$ with $u, v$ positive coprime integers, we have $u \leq r(b+1)$ where $b=\max \left\{\operatorname{dim} f^{-1}(s), s \in \mathcal{S}\right\}$.

(0.2)Theorem([BS1, (0.2.3)]). Let $T$ be the locus of terminal singularities on $M$. Then $\operatorname{cod}_{M} T \geq 3$.

(0.3)Special varieties([BS1] $)$. Let $M$ be an $n$-dimensional normal projective variety with Gorenstein singularities. We say that $(M, L)$ is a Del Pezzo variety (resp. a Mukai variety) if $K_{M} \approx-(n-1) L$ (resp. $\left.K_{M} \approx-(n-2) L\right) ;(M, L)$ is a scroll (resp. a quadric variety) if $K_{M} \approx-(n-1) L$ (resp. $\left.K_{M} \approx-(n-2) L\right) ;(M, L)$ is a scroll (resp. a quadric fibration; resp. a Del Pezzo fibration; resp. a Mukai fibration) over a normal variety $X$ of dimension $m$ if there exists a surjective morphism with connected fibers $\varphi: M \rightarrow L$ such that $K_{M}+(n-m+1) L \approx \varphi^{*} H$ (resp. $K_{M}+(n-m) L \approx \varphi^{*} H$; resp. $\left.K_{M}+(n-m-1) L \approx \varphi^{*} H\right)$ for some ample line bundle $H$ on $X$. Here $\approx$ denotes the linearly equivalence relation.

(0.4)Kobayashi-Ochiai Theorem([F1, $(2,2),(2.3)])$. Let $M$ be an n-dimensional normal projective variety and let $L$ be an ample line bundle on $M$. Then

(i) $(M, L) \cong\left(\mathbb{P}^{n}, O_{\mathbb{P}^{n}}(1)\right)$ if $K_{M}+(n+1) L \sim O_{M}$.

(ii) $(M, L) \cong\left(Q^{n}, O_{Q^{n}}(1)\right), Q^{n}$ is a hyperquadric in $\mathbb{P}^{n+1}$, if $K_{M}+n L \sim O_{M}$. Here $\sim$ denotes the numerically equivalence relation.

(0.5)Theorem([BS1, (2.3)]). Let $M$ be an n-dimensional irreducible normal projective variety with terminal singularities and let $L$ be an ample line bundle on $M$. Assume that $X$ is $\mathbb{Q}$-factorial and that $K_{M}+n L$ is nef and big. Then $K_{M}+n L$ is ample.

(0.6)Theorem(Ma (2.1)]). Let $M$ be an $n$-dimensional normal projective variety with only terminal singularities. Let $L$ be an ample line bundle on $M$. Then $K_{M}+n L$ is nef unless $(M, L) \cong\left(\mathbb{P}^{n}, O_{\mathbb{P}^{n}}(1)\right)$. In particular, $K_{M}+(n+1) L$ is always nef and is ample unless $(M, L) \cong\left(\mathbb{P}^{n}, O_{\mathbb{P}^{n}}(1)\right)$.

(0.7)Theorem([BS1, (0.8.3)]). A rational number $\tau$ is the nef value of $(M, L)$ if and only if $K_{M}+\tau L$ is nef but not ample.

\section{Classifications of polarized varieties}

The following lemma is needed in the sequel.

(1.1)Lemma. Let $M$ be an $n$-dimensional normal projective variety with Gorenstein, terminal, $\mathbb{Q}$-factorial singularities, and let $L$ be an ample line bundle on $M$. Let $\tau$ be the nef value of $(M, L)$. By the Kawamata rationality theorem, $r=u / v$ with $u, v$ positive coprime integers. Assume that $n-k<\tau<n-k+1$ for some positive integer $k<n$. Then

(i) $n \leq 2 k$, and if $n=2 k$ then $(M, L) \cong\left(\mathbb{P}^{n}, O_{\mathbb{P}^{n}}(2)\right)$;

(ii) $2 \leq v \leq \frac{n}{n-k}$ and $\tau=n-k+\frac{i}{v}$ for some positive integer $i<v$ and $i, v$ coprime. 
Proof. From the assumption, we see that $r=u / v$ is not an integer and thus $v \geq 2$. By the Kawamata rationality theorem, we have that $u \leq n+1$, and thus $v(n-k)<$ $u \leq n+1$ or $v(n-k) \leq n$. It follows that $2 \leq v \leq \frac{n}{n-k}$. Moreover, since $v(n-k)<u<v(n-k)+v$, we get that $\tau=n-k+\frac{i}{v}$ for some positive integer $i \leq v$. Note that $u=v(n-k)+i$. If $i, v$ are not coprime, then $u, v$ are not coprime, a contradiction. Hence we find (1.1), (ii).

Let $\varphi: M \rightarrow X$ be the nef value morphism of $(M, L)$. Suppose $n=2 k$. By the above and the Kawamata rationality theorem, we have

$$
u=v k+i \leq \max _{x \in X}\left\{\operatorname{dim} \varphi^{-1}(x)\right\}+1 \leq 2 k+1 .
$$

Since $v \geq 2$ and $i \geq 1$, it follows that $u=2 k+1$ and $v=2$. Thus $\operatorname{dim} \varphi^{-1}(x)=n$ for some $x \in X$, that is, $\varphi$ contracts $M$ to a point. We have $2 K_{M}+(n+1) L \approx O_{M}$. By [BS1, (0.11)] there exists an ample line bundle $A$ on $M$ such that $K_{M} \approx$ $-(n+1) A$ and $L \approx 2 A$. By the Kobayashi-Ochiai Theorem (0.4), $(M, A) \cong$ $\left(\mathbb{P}^{n}, P_{\mathbb{P}^{n}}(1)\right)$ and thus $(M, L) \cong\left(\mathbb{P}^{n}, O_{\mathbb{P}^{n}}(2)\right)$.

The main results of this paper are as follows.

(1.2)Theorem. Let $M$ be a normal projective variety of dimension $n \geq 5$ with Gorenstein, terminal, $\mathbb{Q}$-factorial singularities. Let $L$ be an ample line bundle on $M$. Let $\tau$ denote the nef value of $(M, L)$ and $\varphi: M \rightarrow X$ the nef value morphism of $(M, L)$, and let $F$ be any general fiber of $\varphi$. Assume that $n-4<\tau<n-3$. Then $(M, L)$ must be one of the following.

(i) $n=8, \tau=\frac{9}{2},(M, L) \cong\left(\mathbb{P}^{8}, O_{\mathbb{P}^{8}}(2)\right)$;

(ii) $n=7, \tau=\frac{7}{2}$, either $(M, L) \cong\left(Q^{7}, O_{Q^{7}}(2)\right)$ or $M$ is a $\mathbb{P}^{6}$-bundle over a curve under $\varphi$ and $\left(F, L_{F}\right) \cong\left(\mathbb{P}^{6}, O_{\mathbb{P}^{6}}(2)\right.$;

(iii) $n=6, \tau=\frac{5}{2}, A:=K_{M}+3 L$ is ample on $M .(M, A)$ is one of the following: a) a Del Pezzo variety and $L \approx 2 A$;

b) a quadric fibration over a curve, and $\left(F, L_{F}\right) \cong\left(Q^{5}, O_{Q^{5}}(2)\right)$;

c) a scroll over a surface, and $\left(F, L_{F}\right) \cong\left(\mathbb{P}^{4}, O_{\mathbb{P}^{4}}(2)\right)$;

d) $2 K_{M}+5 L$ is nef and big, $\varphi$ contracts disjoint divisors $E$ to smooth points, and each $\left(E, L_{E}\right) \cong\left(\mathbb{P}^{5}, O_{\mathbb{P}^{5}}(2)\right)$;

(iii') $n=6, \tau=\frac{7}{3},(M, L) \cong\left(\mathbb{P}^{6}, O_{\mathbb{P}^{6}}(3)\right)$;

(iv) $n=5, \tau$ is either $\frac{6}{5}, \frac{5}{4}, \frac{5}{3}, \frac{4}{3}$, or $\frac{3}{2}$.

For $\tau=\frac{6}{5},(M, L) \cong\left(\mathbb{P}^{5}, O_{\mathbb{P}^{5}}(5)\right)$;

For $\tau=\frac{5}{4},(M, L) \cong\left(Q^{5}, O_{Q^{5}}(4)\right)$, or $(M, A)$ is a scroll over a curve for some ample line bundle $A$ on $M$ and $\left(F, L_{F}\right) \cong\left(\mathbb{P}^{4}, O_{\mathbb{P}^{4}}(4)\right)$;

For $\tau=\frac{5}{3},(M, L) \cong\left(Q^{5}, O_{Q^{5}}(3)\right)$, or $(M, A)$ is a scroll over a curve for some ample line bundle $A$ on $M$ and $\left(F, L_{F}\right) \cong\left(\mathbb{P}^{4}, O_{\mathbb{P}^{4}}(3)\right)$;

For $\tau=\frac{4}{3}, A:=2 K_{M}+3 L$ is ample on $M,(M, A)$ is one of the following:

a) a Del Pezzo variety with $L \approx 3 A$;

b) a quadric fibration over a curve with $\left(F, L_{F}\right) \cong\left(Q^{4}, O_{Q^{4}}(3)\right)$;

c) a scroll over a surface with $\left(F, L_{F}\right) \cong\left(\mathbb{P}^{3}, O_{\mathbb{P}^{3}}(3)\right)$; or

d) $3 K_{M}+4 L$ is nef and big, and $\varphi$ contracts disjoint divisors $E$ to points and each $\left(E, L_{E}\right) \cong\left(\mathbb{P}^{4}, O_{\mathbb{P}^{4}}(3)\right)$.

For $\tau=\frac{3}{2}, A:=K_{M}+2 L$ is an ample line bundle on $M,(M, A)$ is either a Mukai variety with $L \approx 2 A$, a Del Pezzo fibration over a curve, a quadric fibration over a surface, a scroll over a 3-dimensional variety, or $2 K_{M}+3 L$ is 
nef and big, $\varphi$ contracts disjoint divisors $E$ to curves or points, the structure of each $E$ is as follows.

a) if $\varphi(E)$ is a point, then $\left(E, L_{E}\right)$ is $\left(\mathbb{P}^{4}, O_{\mathbb{P}^{4}}(2)\right)$, or $\left(Q^{4}, O_{Q^{4}}(2)\right)$;

b) if $\varphi(E)$ is a curve, then $\left(F, L_{F}\right) \cong\left(\mathbb{P}^{3}, O_{\mathbb{P}^{3}}(2)\right)$.

Proof. By Lemma (1.1), $n \leq 8$ and when $n=8,(M, L) \cong\left(\mathbb{P}^{8}, O_{\mathbb{P}^{8}}(2)\right)$. Moreover, from the proof of Lemma (1.1), we have $\tau=\frac{9}{2}$, as in (1.2) (i).

(ii) Let $n=7$. By Lemma (1.1), $2 \leq v \leq \frac{7}{3}$. Thus $v=2$ and by Lemma (1.1) again $\tau=7-4+\frac{1}{2}=\frac{7}{2}$, and so $u=7$. By Kawamata Rationality Theorem (0.1), we have $7 \leq \max _{x \in X}\left\{\operatorname{dim} \varphi^{-1}(x)\right\}+1 \leq 8$ or $6 \leq \max _{x \in X}\left\{\operatorname{dim} \varphi^{-1}(x)\right\} \leq 7$.

Suppose $\max _{x \in X}\left\{\operatorname{dim} \varphi^{-1}(x)\right\}=7$; then $\varphi$ contracts $M$ to a point, and we have $2 K_{M}+7 L \approx O_{M}$. By [BS1, (0.11)], there exists an ample line bundle $A$ on $M$ such that $K_{M} \approx-7 A$ and $L \approx 2 A$. By the Kobayashi-Ochiai Theorem (0.4), we get that $(M, A) \cong\left(Q^{7}, O_{Q^{7}}(1)\right)$. Hence $(M, L) \cong\left(Q^{7}, O_{Q^{7}}(2)\right)$.

Suppose $\max _{x \in X}\left\{\operatorname{dim} \varphi^{-1}(x)\right\}=6$; then $\operatorname{dim} X \geq 1$. We claim that $\operatorname{dim} X=1$.

Indeed, otherwise, assume that $d:=\operatorname{dim} X \geq 2$. First, let $d<n$. Let $F$ be a general fiber of $\varphi: M \rightarrow X$. Then $\operatorname{dim} F \leq \overline{5}$. Since $\tau=\frac{7}{2}$, we have $2 K_{F}+$ $7 L_{F} \approx O_{F}$. By [BS1 (0.11)], there exists an ample line bundle $A$ on $F$ such that $K_{F}+7 A \approx O_{F}$ and $7 \leq \operatorname{dim} F+1 \leq 6$, a contradiction. Second, let $d=n$. Then $2 K_{M}+7 L$ is nef and big. Write $K_{M}+7\left(K_{M}+4 L\right)=4\left(2 K_{M}+7 L\right)$. Then $A:=K_{M}+4 L$ is an ample line bundle on $M$, and $K_{M}+7 A$ is nef and big but not ample. However, by Theorem (0.5),$K_{M}+7 A$ is ample. We get a contradiction.

Now, we have that $\operatorname{cod}_{M} \operatorname{Sing}(M) \geq 3>\operatorname{dim} X$ by Theorem (0.2) and that $u=\max _{x \in X}\left\{\operatorname{dim} \varphi^{-1}(x)\right\}+1=7$. By BS2 (1.4)], $M$ is a $\mathbb{P}^{6}$-bundle over a curve $X$ under $\varphi$. Finally, for any general fiber $F$ of $\varphi$, we have $2 K_{F}+7 L_{F} \approx O_{F}$. Note that $F \cong \mathbb{P}^{6}$ and $K_{M} \cong O_{\mathbb{P}^{6}}(-7)$. We get that $\left(F, L_{F}\right) \cong\left(\mathbb{P}^{6}, O_{\mathbb{P}^{6}}(2)\right.$, as in (1.2) (ii).

(iii) Let $n=6$. By Lemma (1.1), $2 \leq v \leq \frac{6}{6-4}=3$, so $v=2$ or $v=3$.

Let $v=2$ and $\tau=\frac{u}{v}=6-4+\frac{1}{2}=\frac{5}{2}$. Write $K_{M}+5\left(K_{M}+3 L\right)=3\left(2 K_{M}+5 L\right)$. Then $A:=K_{M}+3 L$ is an ample line bundle on $M$.

First, assume that $\varphi: M \rightarrow X$ has lower dimensional image. If $\operatorname{dim} X=0$, then $K_{M}+5 A \approx O_{M}$ and $L \approx 2 A$. By definition (0.3), $(M, A)$ is a Del Pezzo variety.

Let $\operatorname{dim} X \geq 1$, and let $F$ be any general fiber of $\varphi$. Since $2 K_{M}+5 L_{M} \approx \varphi^{*} H$ for some ample line bundle $H$ on $X$ by [KMM, $(3-2-1)], K_{M}+5 A \approx \varphi^{*}(3 H)$.

If $\operatorname{dim} X=1,(M, A)$ is a quadric fibration over $X$ under $\varphi$ by definition. Moreover, $K_{F}+5 A_{F} \approx O_{F}$. Since $\operatorname{dim} F=5,\left(F, A_{F}\right) \cong\left(Q^{5}, O_{Q^{5}}(1)\right)$ by (0.4) F1. Note that $K_{F} \cong O_{Q^{5}}(-t)$ and $2 K_{F}+5 L_{F} \cong O_{F}$. We get $\left(F, L_{F}\right) \cong\left(Q^{5}, O_{Q^{5}}(2)\right)$.

If $\operatorname{dim} X=2,(M, A)$ is a scroll over $X$ under $\varphi$ since $K_{M}+5 A \approx \varphi^{*}(3 H)$. Note that $\operatorname{dim} F=4$ and $K_{F}+5 A_{F} \approx O_{F}$. We have $\left(F, A_{F}\right) \cong\left(P^{4}, O_{\mathbb{P}^{4}}(1)\right)$. Since $K_{F} \cong O_{\mathbb{P}^{4}}(-5)$ and $2 K_{F}+5 L_{F} \cong O_{F}, L_{F} \cong O_{\mathbb{P}^{4}}(2)$ and so $\left(F, L_{F}\right) \cong\left(\mathbb{P}^{4}, O_{\mathbb{P}^{4}}(2)\right)$.

If $\operatorname{dim} X \geq 3$, then $\operatorname{dim} F \leq 3$. Since $K_{F}+5 A_{F} \approx O_{F}, K_{F}+5 A_{F}$ is ample on $F$ by Theorem (0.6). We get a contradiction. Therefore, $\operatorname{dim} X \leq 2$.

Second, assume that $\varphi: M \rightarrow X$ is birational. Then $K_{M}+5 A$ is nef and big but not ample. By Theorem (0.7) the nef value of $(M, A)$ is 5 . By [An, Theorem 1], $\varphi$ contracts disjoint divisors $E \cong \mathbb{P}^{5}$ to smooth points. Moreover, $L_{E}(E) \cong O_{\mathbb{P}^{5}}(-1)$ and $A_{E} \cong O_{\mathbb{P}^{5}}(1)$. Note that $\left.A_{E} \approx K_{M}\right|_{E}+3 L_{E}$ and $\left.K_{M}\right|_{E}+O_{E}(E) \cong K_{E} \cong$ $O_{\mathbb{P}^{5}}(-6)$. We find that $L_{E} \cong O_{\mathbb{P}^{5}}(2)$. Therefore, $\left(E, L_{E}\right) \cong\left(\mathbb{P}^{5}, O_{\mathbb{P}^{5}}(2)\right)$.

(iii') Let $n=6$ and $v=3$. Note that $2<\tau=\frac{u}{3}<3$ and $u \leq 7$. We have $\tau=\frac{7}{3}$. Then $u=7 \leq \max _{x \in X}\left\{\operatorname{dim} \varphi^{-1}(x)\right\}+1 \leq 7$ by (10.1), and so 
$\max _{x \in X}\left\{\operatorname{dim} \varphi^{-1}(x)\right\}=6$. It follows that $\varphi$ contracts $M$ to a point. Thus $3 K_{M}+$ $7 L \approx O_{M}$. Let $A=2 K_{M}+5 L$, then $K_{M}+7 A=5\left(3 K_{M}+7 L\right) \approx O_{M}$. Thus, by $(0.4),(M, A) \cong\left(\mathbb{P}^{6}, O_{\mathbb{P}^{6}}(1)\right)$. Since $K_{M} \approx O_{\mathbb{P}^{6}}(-7)$, we find that $(M, L) \cong$ $\left(\mathbb{P}^{6}, O_{\mathbb{P}^{6}}(3)\right)$.

(iv) Let $n=5$. By Lemma (1.1) and the Kawamata Rationality Theorem (0.1), we have that $2 \leq v \leq \frac{5}{5-4}=5,1<\tau=1+\frac{i}{v}<2$, and $u \leq \max _{x \in X}\left\{\operatorname{dim} \varphi^{-1}(x)\right\}+$ $1 \leq 6$. It follows that $\tau$ is either $\frac{6}{5}, \frac{5}{4}, \frac{5}{3}, \frac{4}{3}$, or $\frac{3}{2}$.

For $\tau=\frac{6}{5}$, we have $\max _{x \in X}\left\{\operatorname{dim} \varphi^{-1}(x)\right\}+1=6$ and so $\varphi$ contracts $M$ to a point. Thus $5 K_{M}+6 L \approx O_{M}$. Write $K_{M}+6\left(4 K_{M}+5 L\right)=5\left(5 K_{M}+6 L\right)$. Then $A:=4 K_{M}+5 L$ is an ample line bundle on $M$, and $K_{M}+6 A \approx O_{M}$. By (0.4) [F1] we have $(M, A) \cong\left(\mathbb{P}^{5}, O_{\mathbb{P}^{5}}(1)\right)$. Clearly, $L \cong O_{\mathbb{P}^{5}}(5)$ and hence $(M, L) \cong\left(\mathbb{P}^{5}, O_{\mathbb{P}^{5}}(5)\right)$.

For $\tau=\frac{5}{4}$, there exists an ample line bundle $H$ such that $4 K_{M}+5 L \approx \varphi^{*} H$. Write $K_{M}+5\left(3 K_{M}+4 L\right)=4\left(4 K_{M}+5 L\right)$. Then $A:=3 K_{M}+4 L$ is an ample line bundle on $M$ and $K_{M}+5 A \approx \varphi^{*}(4 H)$.

First, assume that $\varphi$ has lower dimensional image. If $X$ is a point, then $K_{M}+$ $5 A \approx O_{M}$ and thus $(M, A) \cong\left(Q^{5}, O_{Q^{5}}(1)\right.$ by (0.4) [F1]. From $A=3 K_{M}+4 L$, we find that $L \cong O_{Q^{5}}(4)$. Therefore, $(M, L) \cong\left(Q^{5}, O_{Q^{5}}(4)\right)$.

If $\operatorname{dim} X=1$, then $(M, A)$ is a scroll over $X$ under $\varphi$ since $K_{M}+5 A \approx \varphi^{*}(4 H)$. Note that $K_{F}+5 A_{F} \approx O_{F}$. We have $\left(F, A_{F}\right) \cong\left(\mathbb{P}^{4}, O_{\mathbb{P}^{4}}(1)\right)$. Clearly, $L_{F} \cong O_{\mathbb{P}^{4}}(4)$.

We claim that $\operatorname{dim} X \leq 1$. Indeed, otherwise, let $\operatorname{dim} X \geq 2$; then $\operatorname{dim} F \leq 3$ and $K_{F}+5 A_{F} \approx O_{F}$. But, by Theorem (0.6), $K_{F}+5 A_{F}$ is ample on $F$, a contradiction.

Second, assume that $\varphi: M \rightarrow X$ is birational. Then $K_{M}+5 A$ is nef and big but not ample. But, by Theorem (0.5), $K_{M}+5 A$ is ample. We get a contradiction. This shows that $\varphi$ cannot be birational.

Similarly, for $\tau=\frac{5}{3}$, we have that $(M, L) \cong\left(Q^{5}, O_{Q^{5}}(3)\right)$ or $(M, A)$ is a scroll over a curve under $\varphi$, and $\left(F, L_{F}\right) \cong\left(\mathbb{P}^{4}, O_{\mathbb{P}^{4}}(3)\right)$, where $A:=K_{M}+2 L$ is an ample line bundle on $M$.

For $\tau=\frac{4}{3}, K_{M}+4\left(2 K_{M}+3 L\right)=3\left(3 K_{M}+4 L\right) \approx \varphi^{*}(3 H)$ for some ample line bundle $H$ on $X . A:=2 K_{M}+3 L$ is an ample line bundle on $M$. When $\varphi$ has lower dimensional image, from $K_{M}+4 A \approx \varphi^{*}(3 H)$, we have that $(M, A)$ is either a Del Pezzo variety with $L \approx 3 A$, a quadric fibration over a curve with $\left(F, L_{F}\right) \cong\left(Q^{4}, Q_{Q^{4}}(3)\right)$, or a scroll over a surface with $\left(F, L_{F}\right) \cong\left(\mathbb{P}^{3}, O_{\mathbb{P}^{3}}(3)\right)$. When $\varphi$ is birational, then $K_{M}+4 A$ is nef and big but not ample, by $\underline{\text { An }}$, Theorem 1] $\varphi$ contracts disjoint divisors $E$ to points. Moreover, $\left(E, A_{E}\right) \cong\left(\mathbb{P}^{4}, O_{\mathbb{P}^{4}}(1)\right)$ and $O_{E}(E) \cong O_{\mathbb{P}^{4}}(-1)$. Thus $\left(E, L_{E}\right) \cong\left(\mathbb{P}^{4}, O_{\mathbb{P}^{4}}(3)\right)$.

For $\tau=\frac{3}{2}$, write $K_{M}+3\left(K_{M}+2 L\right)=2\left(2 K_{M}+3 L\right)$. Then $A:=K_{M}+2 L$ is ample on $M$. Since $2 K_{M}+3 L \approx \varphi^{*} H$ for some line bundle $H$ on $X, K_{M}+3 A \approx \varphi^{*}(2 H)$. When $\varphi$ has lower dimensional image, we get that $(M, A)$ is either a Mukai variety with $L \approx 2 L$, a Del Pezzo fibration over a curve, a quadric fibration over a surface, or a scroll over a 3 -dimensional variety.

When $\varphi$ is birational, then $K_{M}+3 A$ is nef and big but not ample. Let $E$ be the exceptional locus of $\varphi$. By [An, Theorem 3] and its proof, we get that $\varphi$ contracts disjoint divisors $E$ to curves or points. Moreover, if $\varphi(E)$ is a point, then $\left(E, L_{E}\right)$ is $\left(\mathbb{P}^{4}, O_{\mathbb{P}^{4}}(2)\right)$, or $\left(Q^{4}, O_{Q^{4}}(2)\right)$. If $\varphi(E)$ is a curve, then $\left(F, L_{F}\right) \cong\left(\mathbb{P}^{3}, O_{\mathbb{P}^{3}}(2)\right)$.

(1.3)Theorem. Let $M$ be a normal projective variety of dimension $n \geq 4$ with Gorenstein, terminal, $\mathbb{Q}$-factorial singularities. Let $L$ be an ample line bundle on $M$. Let $\tau$ be the nef value of $(M, L)$ and $\varphi: M \rightarrow X$ the nef value morphism of 
$(M, L)$. Let $F$ be any general fiber of $\varphi$. Assume that $n-3<\tau<n-2$. Then $(M, L)$ must satisfy one of the following:

(i) $n=6, \tau=\frac{7}{2},(M, L) \cong\left(\mathbb{P}^{6}, O_{\mathbb{P}^{6}}(2)\right)$;

(ii) $n=5, \tau=\frac{5}{2}, A:=K_{M}+3 L$ is ample on $M$, either $(M, L) \cong\left(Q^{5}, O_{Q^{5}}(2)\right)$ or $(M, A)$ is a scroll over a curve under $\varphi$ and $\left(F, L_{F}\right) \cong\left(\mathbb{P}^{4}, O_{\mathbb{P}^{4}}(2)\right)$;

(iii) $n=4, \tau=\frac{5}{3}, \frac{5}{4}, \frac{4}{3}$, or $\frac{3}{2}$.

For $\tau=\frac{5}{3},(M, L) \cong\left(\mathbb{P}^{4}, O_{\mathbb{P}^{4}}(3)\right)$;

For $\tau=\frac{5}{4},(M, L) \cong\left(\mathbb{P}^{4}, O_{\mathbb{P}^{4}}(4)\right)$;

For $\tau=\frac{4}{3}, A:=2 K_{M}+3 L$ is ample on $M$, either $(M, L) \cong\left(Q^{4}, O_{Q^{4}}(3)\right)$ or $(M, A)$ is a scroll over a curve and $\left(F, L_{F}\right) \cong\left(\mathbb{P}^{3}, O_{\mathbb{P}^{3}}(3)\right)$.

For $\tau=\frac{3}{2}, A:=K_{M}+2 L$ is ample on $M,(M, A)$ is one of the following:

a) A Del Pezzo variety;

b) a quadric fibration over a curve;

c) a scroll over a surface; or

d) $2 K_{M}+3 L$ is nef and big, $\varphi$ contracts disjoint divisors $E$ to smooth points, and $\left(E, L_{E}\right) \cong\left(\mathbb{P}^{3}, O_{\mathbb{P}^{3}}(2)\right)$.

Proof. By Lemma (1.1) we have $n \leq 6$.

(i) Let $n=6$. Then $v=2, \tau=6-3+\frac{1}{2}=\frac{7}{2}$, and $(M, L) \cong\left(\mathbb{P}^{6}, O_{\mathbb{P}^{6}}(2)\right)$.

(ii) Let $n=5$. Then $2 \leq v \leq \frac{t}{5-2}=\frac{5}{2}$ and thus $v=2, \tau=5-3+\frac{1}{2}=\frac{5}{2}$. Write $K_{M}+5\left(K_{M}+3 L\right)+3\left(2 K_{M}+5 L\right)$. Then $A:=K_{M}+3 L$ is an ample line bundle on $M$. There exists an ample line bundle $H$ on $X$ such that $K_{M}+5 A \approx \varphi^{*}(3 H)$.

First, assume that $\varphi$ has lower dimensional image. Let $F$ be any general fiber of $\varphi$. If $X$ is a point, then $(M, A) \cong\left(Q^{5}, O_{Q^{5}}(1)\right)$ by 0.4 . Thus $(M, L) \cong$ $\left(Q^{5}, O_{Q^{5}}(2)\right)$.

If $\operatorname{dim} X=1$, then $(M, A)$ is a scroll over a curve under $\varphi$ by definition, and $\left(F, L_{F}\right) \cong\left(\mathbb{P}^{4}, O_{\mathbb{P}^{4}}(2)\right)$.

If $\operatorname{dim} X \geq 2$, then $\operatorname{dim} F \leq 3$ and $K_{F}+5 A_{F} \approx O_{F}$. But by $\left(\underline{0.6}, K_{F}+5 A_{F}\right.$ is ample on $F$. We get a contradiction.

Second, assume that $\varphi$ is birational. Then $K_{M}+5 A$ is nef and big but not ample. But by (0.5), $K_{M}+5 A$ is ample, a contradiction. Thus, $\varphi$ cannot be birational.

(iii) Let $n=4$. Then $1<\tau=\frac{u}{v}<2$ and $u \leq 5$. Using Lemma (1.1), we find that $\tau$ is either $\frac{5}{3}, \frac{5}{4}, \frac{4}{3}$, or $\frac{3}{2}$.

For $\tau=\frac{5}{3}$, write $K_{M}+5\left(K_{M}+2 L\right)=2\left(3 K_{M}+5 L\right)$ and $A:=K_{M}+2 L$ is ample on $M$. There exists an ample line bundle $H$ on $X$ such that $K_{M}+5 A \approx \varphi^{*}(2 H)$.

First, assume that $\varphi$ has lower dimensional image. If $X$ is a point, then $(M, A) \cong$ $\left(\mathbb{P}^{4}, O_{\mathbb{P}^{4}}(1)\right)$. Clearly, $L \cong O_{\mathbb{P}^{4}}(3)$. Hence $(M, L) \cong\left(\mathbb{P}^{4}, O_{\mathbb{P}^{4}}(3)\right)$.

If $\operatorname{dim} X \geq 1$, then $\operatorname{dim} F \leq 3$ and $K_{F}+5 A_{F} \approx O_{F}$. But by (0.6), $K_{F}+5 A_{F}$ is ample on $F$. We get a contradiction.

Second, assume that $\varphi$ is birational. Then $K_{M}+5 A$ is nef and big but not ample. But by (0.5), $K_{M}+5 A$ is ample, a contradiction. Thus, $\varphi$ cannot be birational.

Similarly, for $\tau=\frac{5}{4}$, we have $(M, L) \cong\left(\mathbb{P}^{4}, O_{\mathbb{P}^{4}}(4)\right)$.

For $\tau=\frac{4}{3}, K_{M}+4\left(2 K_{M}+3 L\right)=3\left(3 K_{M}+4 L\right) \approx \varphi^{*}(3 H)$ for some ample line bundle $H$ on $X . A:=2 k_{M}+3 L$ is an ample line bundle on $M$. By the same way as above, we get that either $(M, L) \cong\left(Q^{4}, O_{Q^{4}}(3)\right)$ or $(M, A)$ is a scroll over a curve and $\left(F, L_{F}\right) \cong\left(\mathbb{P}^{3}, O_{\mathbb{P}^{3}}(3)\right)$.

For $\tau=\frac{3}{2}$, write $K_{M}+3\left(K_{M}+2 L\right)=2\left(2 K_{M}+3 L\right)$. Then $A:=K_{M}+2 L$ is ample on $M$ and $K_{M}+3 A \approx \varphi^{*}(2 H)$ for some line bundle $Y$ on $X$. When $\varphi$ 
has lower dimensional image, we get that $(M, A)$ is either a Del Pezzo variety with $L \approx 2 L$, a quadric fibration over a curve with $\left(F, L_{F}\right) \cong\left(Q^{3}, O_{Q^{3}}(2)\right)$, or a scroll over a surface with $\left(F, L_{F}\right) \cong\left(\mathbb{P}^{2}, O_{\mathbb{P}^{2}}(2)\right)$.

When $\varphi$ is birational, $K_{M}+3 A$ is nef and big but not ample. By [An, Theorem 1], $\varphi$ contracts disjoint divisors $E$ to smooth points. Moreover, $\left.O_{E}(E) \cong O_{\mathbb{P}^{3}}(-1)\right)$ and $\left(E, A_{E}\right) \cong\left(\mathbb{P}^{3}, O_{\mathbb{P}^{3}}(1)\right)$. Hence $\left(E, L_{E}\right) \cong\left(\mathbb{P}^{3}, O_{\mathbb{P}^{3}}(2)\right)$. The proof is completed.

\section{REFERENCES}

[An] M. Andreatta, Contractions of Gorenstein polarized varieties with high nef value, Math. Ann. 300 (1994), 669-679. MR 96b:14007

[BS1] M. Beltrametti and J. A. Sommese, On the adjunction theoretic classification of polarized varieties, J. Reine. Angew. Math. 427 (1992), 157-192. MR 93d:14012

[BS2] M. Beltrametti and J. A. Sommese, A remark on the Kawamata rationality theorem, J. Math. Soc. Japan 45 (1993), 557-568. MR 94e:14006

[F1] T. Fujita, Remarks on quasi-polarized varieties, Nagoya Math. J. 115 (1989), 105-123. MR 90i:14045

[F2] T. Fujita, On Kodaira energy and adjoint reduction of polarized manifolds, Manuscr. Math. 75 (1992), 59-84. MR 93i:14032

[KMM] Y. Kawamata, K. Matsuda, K. Matsuki, Introduction to the Minimal Model Program, Advanced Studies in Pure Math. 10 (1987), 283-360. MR 89e:14015

[Ma] H. Maeda, Ramification divisors for branched coverings of $\mathbb{P}^{n}$, Math. Ann. 288 (1990), 195-199. MR 91i:14011

[Mo] S. Mori, Threefolds whose canonical bundles are not numerically effective, Ann. Math. 116 (1982), 133-176. MR 84e:14032

[S] A. J. Sommese, On the adjunction theoretic structure of projective varieties, Lecture Notes Math. 1194 (1984), 175-213. MR 87m:14049

Department of Mathematics, Jinan University, Guangzhou 510632, People's Republic OF CHINA 